\title{
Kein trockener Anfängerunterricht mehr! Eine dramapädagogische Modelleinheit für die ersten DaF-Stunden
}

\author{
Ulrich Wettemann
}

\begin{abstract}
Zusammenfassung
Der dramapädagogische Ansatz wird bis dato primär bei fortgeschrittenen Lernern eingesetzt. Dass und wie dieser Ansatz auch bei Sprachanfängern nützlich angewendet werden kann, möchte ich im Folgenden darstellen. Dafür stelle ich zuerst die erwarteten Kann-Beschreibungen heraus. Als weitere theoretische Grundlage diskutiere ich dann wichtige Hypothesen des Spracherwerbs und setze diese mit dem Konzept autonomen Lernens in Verbindung. Auf dieser theoretischen Grundlage erörtere ich daraufhin die Übertragbarkeit des dramapädagogischen Ansatzes auf den DaF-Anfängerunterricht. Für die Entwicklung von geschlossenen und halboffenen Übungsformen prüfe ich das Kontinuum von Process Drama und erläutere, wie der Ansatz durch die Verknüpfung mit dem autonomen Lernen schon ab der ersten Unterrichtsstunde eingesetzt werden kann, um einen systematischen Erwerb ${ }^{1}$, von Sprachflüssigkeit zu ermöglichen. Abschließend wird die Anwendung in einem dreiwöchigen Intensivkurs für Sprachanfänger in Mexiko ausgewertet. Auf der Basis von Videoaufzeichnungen und eines offenen Evaluationsbogens zeigt sich, dass die Kombination beider Ansätze zu einem schnellen Spracherfolg und zur Aufrechterhaltung der Motivation führt.
\end{abstract}

\section{Forschungsstand und Problemstellung}

Grundlage des dramapädagogischen Ansatzes ${ }^{2}$ bildet das kommunikative Fremdsprachenlehren und -lernen. Mit Hilfe dramatischer Kunstformen werden den Teilnehmern Lernformen eröffnet, die durch Spannungsgeladenheit zum spontanen kommunikativen Handeln einladen „to reproduce in their own words what they have learned through receptive skills before" (Shimizu 1993: 145f).

\footnotetext{
${ }^{1}$ Erlernen und Erwerben wird in diesem Artikel als Synonym verwendet.

2 Obwohl Schewe (1993) und Ortner (1998) von einer Drama-Methode sprechen und Schewe (2000:72) zu dem Begriff „Konzept eines dramapädagogischen Fremdsprachenunterrichts“ wechselt, möchte ich im Folgenden von einem „dramapädagogischen Ansatz“ sprechen, um seine Offenheit und Prozesshaftigkeit hervorzuheben.
} 
Die komplexen Aktivitäten, die in einen fiktiven Situationsrahmen eingebettet sind, beanspruchen das intellektuelle, motorische, affektive und interaktive Engagement der Lernenden. Improvisation gilt als zentraler Faktor, wodurch den Teilnehmern die Freiheit gegeben wird, sich kreativ und persönlich einbringen zu können (vgl. Schewe 1993). Der dramapädagogische Ansatz gewinnt in der Fremdsprachendidaktik immer mehr an Bedeutung, was sich in der positiven Resonanz auf die für fortgeschrittene Lerner entwickelten Unterrichtsmodelle (vgl. Even 2003, besonders Kapitel VII) widerspiegelt.

Ziel dieses Artikels ist es erste Ansätze zu entwickeln, mit denen der dramapädagogische Ansatz auch für Sprachanfänger (ohne Vorkenntnisse) gewinnbringend eingesetzt werden kann. Meines Wissens wurde die von Schewe (1993: 417) aufgeworfene Problemstellung „Für Fortgeschrittene mag dieses Konzept geeignet sein, aber was mache ich in Anfängergruppen?" bis dato nämlich nicht weiter geklärt (Graz: IDT 2005. Schewe: Dramapädagogik in Forschung und Lehre - Blick zurück nach vorn). Wie könnte also die Dramapädagogik für Sprachanfänger umgedacht oder erweitert werden, da von ihnen kein spontanes Sprechen erwartet werden kann? Was sollen Anfänger überhaupt leisten können? Als Orientierungshilfe möchte ich die globalen KannBeschreibungen aus Profile Deutsch (2002) zur Hand nehmen. Hier tritt die Zielsetzung „spontan sprechen zu können“ zum ersten Mal in der mündlichen Interaktion in der Niveaustufe B2 auf, wobei jedoch die Rahmensetzung ,in vertrauten Situationen "immer noch beibehalten wird“ (ibid. 119ff). Der Lerner
kann sich so spontan und fließend verständigen, dass ein normales Ge- spräch mit deutschsprachigen Gesprächspartnern ohne größere Anstren- gung auf beiden Seiten gut möglich ist und kann dabei Fehler, die zu Missverständnissen geführt haben, meist selbst korrigieren. (Hervorhe- bung U.W.)

Am Ende der Niveaustufe A1 wird aber vorerst nur erwartet, dass der Sprachlerner

mit wenigen, einfachen und auswendig gelernten Ausdrücken und Sätzen vertraute Situationen bewältigen kann, die ganz alltägliche und konkrete Bedürfnisse betreffen, wobei es zu Missverständnissen kommen kann. (ibid. 75)

Um den dramapädagogischen Ansatz schon bei Sprachanfängern nützlich anwenden zu können, muss die Ausgestaltung eines dramapädagogischen Sprachunterrichts für Lernanfänger eine Modifizierung erfahren.

\section{Fragestellung an die Fremdsprachenlehr- und-lernforschung}

Zur Klärung dieser Modifizierung möchte ich von den multiplen Faktoren des Fremdsprachenunterrichts die tragenden Aspekte diskutieren, die ich 
für ein dramapädagogisches Unterrichtsarrangement bei Sprachanfängern für besonders wichtig halte: Diese umfassen einerseits die Frage nach dem Input, der bei Sprachanfängern vorrangig von außen kommen muss und andererseits die Frage nach dem Output und dessen Bedeutung. Zwischen diesen beiden Polen befindet sich die spannende und relativ ungeklärte Frage nach der Sprachverarbeitung ${ }^{3}$, d.h. wie kann Input zu Intake werden? Welche Schritte müssen also stattgefunden haben, bis eine sprachliche Anwendung in Interaktion erfolgen kann? (vgl. Tönshoff 1995: 9ff)

\subsection{Input}

Jeder Sprachlernprozess beginnt mit Input. Wenn der Lerner diesen Input verstanden und innerlich verarbeitet hat, wird er als Intake bezeichnet. Krashen (1982) geht davon aus, dass Sprache durch verständlichen Input erworben wird und sich die Sprachproduktion dann von selbst entwickelt. Woher kommt jedoch dieser Input? Für die dramapädagogische Arbeitsweise ist es charakteristisch, „dass der Lerner-Input zum eigentlichen Input wird“ (Schewe 1993: 348f, siehe auch Kao/O'Neill 1998: 15), was für fortgeschrittene Lerner auch plausibel erscheint. Bei Sprachanfängern kann jedoch nicht nach dieser Arbeitsweise verfahren werden, da sie noch kaum eigenen Input beitragen können. Damit sie also mit wenigen, einfachen und auswendig gelernten Ausdrücken und Sätzen vertraute Situationen bewältigen können (vgl. Kapitel 1), muss ihnen ein qualitativer Input zur Verfügung gestellt werden. Für die Zusammenstellung in meiner Modelleinheit orientiere ich mich vorrangig an dem Lehrwerk Berliner Platz $A 1^{4}$ (2002) und an Profile Deutsch (2002) und versuche, auf dem Boden meiner langjährigen Erfahrung in Mexiko, diesen Input an die Bedürfnisse meiner Adressaten anzupassen. Gleichzeitig schaffe ich Unterrichts-Freiräume, damit sich die Lerner mit Vorschlägen ${ }^{5}$ aus ihrer Muttersprache, die ich dann pragmatisch angemessen ins Deutsche übertrage, zunehmend einbringen können. ${ }^{6}$

\footnotetext{
${ }^{3}$ Even (2003) hat zwar durch ,Dramagrammatik' erste Ansätze unternommen, den Verarbeitungsprozess weiter in den Mittelpunkt zu rücken (siehe insbesondere Kapitel 3.3), bezieht sich in ihren Umsetzungsmodellen jedoch nur auf fortgeschrittene Lerner, wobei sie bekannte grammatische Phänomene mit ihnen wiederholt und vertieft. Das Sprachwissen wird bei den Lernern größtenteils vorausgesetzt. Bei Anfängern können diese Unterrichtsphasen folglich nicht angewendet werden.

${ }^{4}$ Dieses Lehrwerk wurde von der Sprachschule Centro de Idiomas als Folgelehrwerk von Themen neu ausgewählt und führt alle Teilnehmer bis zur Prüfung: Zertifikat Deutsch.

${ }^{5}$ Vielseitige Beispiele sind in Anhang B: 1.-6. zu finden und insbesondere in Fußnote 29.

6 Inwieweit Lerner sich in den Unterrichtsverlauf einbringen und dadurch Verantwortung übernehmen können, wird u.a. durch die Öffnung von Unterricht und einer veränderten Lehrerrolle ermöglicht. In Absatz 3 werde ich ausführlicher auf das so genannte autonome Lernen eingehen.
} 


\subsection{Interaktion und Output}

Mit dem Bereitstellen von angemessenem Input ist zwar ein erster Schritt für die Adaption des dramapädagogischen Ansatzes bei Sprachanfängern unternommen. Jedoch, so führt Ortner (1998: 148) aus, schränkt sich die Methode auf die Anwendung in der Interaktion ein, da der Weg vom Input über die Sprachaufnahme bis zur Anwendung nicht nachgezeichnet ist. Welchen Stellenwert schreibe ich also der Interaktion bei Sprachanfängern zu?

In meinem dramapädagogischen Ansatz stimme ich mit der InteraktionsHypothese (Long 1983) darin überein, dass Input zur Sicherstellung von Spracherwerb in Interaktion umgesetzt werden muss. Ich löse mich jedoch von dem Konzept der Bedeutungsaushandlung, mit dem die Interaktions-Hypothese eng verknüpft ist (vgl. Henrici 1995: 14ff), da Sprachanfänger dies einfach noch nicht leisten können. Interaktion wird daher in meinem Ansatz mit der Schaffung von Sprachlern- und Sprachgebrauchssituationen verbunden, in denen authentische Spracherfahrungen gemacht und formal-sprachliche Kompetenzen erworben werden.

Zur Veranschaulichung möchte ich auf die Output ${ }^{7}$-Hypothese von Swain (1985), die die Input- und Interaktions-Hypothese erweitert, etwas genauer eingehen. Swain folgert aus einer Untersuchung von Schülern, die trotz jahrelangem Input durch bilingualen Fachunterricht nur in der Lage waren eine Art „Unterrichtspidgin“ zu sprechen, dass verständlicher Input für den Spracherwerb nicht ausreicht. Ihre Output-Hypothese akzentuiert somit, dass die Lernenden erst durch den Gebrauch dazu geführt werden, Sprache auch formal zu verarbeiten ${ }^{8}$ (vgl. Henrici 1995: 17). Also durch die Anstrengung des Lerners sich auszudrücken, werden vorhandene Kenntnisse aktiviert, vertieft, neu miteinander in Verbindung gebracht und automatisiert (vgl. Edmondson/House 2000: 27). Die Aufforderung zum Sprechen reicht allerdings nicht aus, um die zielsprachlichen Strukturen richtig zu erwerben. Deshalb muss der Lehrer besonders auf korrekten Sprachgebrauch achten. Swain bezeichnet dies als pushed output, worauf der Fokus primär bei L2-Anfängern gerichtet sein muss, damit sie die formal-sprachlichen Kompetenzen angemessen entwickeln können (vgl. Even 2003: 95; Edmondson/House 2000: 279). Gestützt auf diese Hypothese möchte ich an einigen dramapädagogischen Übungen aufzeigen (vgl. Kap. 4.1 und 4.2), wie pushed output kreativ und motivierend für Anfänger umgesetzt werden kann. Auch die allgemeingültigen Kriterien der Sprachverarbeitung, auf die ich im folgenden Absatz genauer eingehen möchte, werden dabei berücksichtigt.

\footnotetext{
7 Unter Output ist sowohl die mündliche als auch schriftliche Sprachproduktion in der Zielsprache zu verstehen. In diesem Artikel geht es jedoch vorrangig um die mündliche Sprachproduktion.

${ }^{8}$ Im Gegensatz zum reinen Verstehen, wo nur inhaltliche Aspekte im Vordergrund stehen.
} 


\subsection{Variablen der Sprachverarbeitung}

Da es bis dato noch keine klare Antwort auf die Frage nach der Sprachverarbeitung gibt (vgl. Edmondson/House 2000: 330), orientiere ich mich bei meiner Modelleinheit an den drei Variablen, die prinzipiell als ausschlaggebend für den Sprachlernprozess angesehen werden: nämlich inwieweit vorhandenes Wissen analysiert, integriert und automatisiert ist (ibid. 323). Analyse, Integration und Automatisierung bilden somit unverzichtbare Elemente der Sprachverarbeitung, die allerdings nicht in dieser Reihenfolge stattfinden müssen. Denn ,Chunks' z.B. werden zuerst als ganze Ausdrücke der Zielsprache gelernt und automatisiert. Eine Analyse kann vom Lerner erst viel später stattfinden, wodurch er sich der Grammatik der Zielsprache annähert (ibid. 279). Die Arbeitsweise meiner Modelleinheit besteht deshalb in Bezug auf Input vorrangig aus der Bereitstellung von Chunks, die in kreativen und ganzheitlichen Übungsformen automatisiert und integriert werden. Eine Analyse findet erst dann statt, wenn es von einzelnen Lernern erwünscht wird bzw. wenn es zur Bewusstmachung von Fehlerquellen beiträgt. Diese Arbeitsweise, so zeigt es sich in der Auswertung der Modelleinheit (vgl. Kap. 5), führt zu einem schnellen Sprachgebrauch und zur Aufrechterhaltung der Motivation. Hinter diesem Unterrichtsarrangement, das auf den ersten Eindruck sehr einfach erscheinen mag, befindet sich jedoch ein komplexer Umsetzungsprozess, der versucht, den Lerner stufenweise zu einer wachsenden Selbststeuerung zu befähigen. Für eine Erfolg versprechende Modifizierung und Implementierung des dramapädagogischen Ansatzes bei Sprachanfängern sehe ich deshalb die Förderung des autonomen Lernens als unverzichtbar an und möchte im folgenden Kapitel genauer darauf eingehen.

\section{Lernerautonomie}

\subsection{Hintergrund und Begriff}

Die Hinwendung zur Lernerautonomie entwickelte sich vor allem durch die Abkehr vom Behaviorismus, der von einem passiven und nachahmenden Lerner ausging. Diese Vorstellung wurde durch die kognitive Lerntheorie abgelöst, die den Lerner in den Mittelpunkt des Lehr- und Lernprozesses rückt. Lernerorientierung bedeutet demnach den Lerner als Subjekt des Unterrichts zu betrachten und dessen Verstehens- und Mitteilungsbedürfnisse zu fördern (vgl. Düwell 2003: 350).

$\mathrm{Zu}$ den ersten und bedeutendsten Vertretern der Lernerautonomie zählt zweifellos Holec (1979), der Lernerautonomie folgendermaßen definiert:

[...] autonomy is ,the ability to assume responsibility for one's own affairs". In the context which we are dealing, the learning of languages, autonomy is consequently the ability to take charge of one's own learning. (ibid. 3; Hervorhebung im Original)

Unter „ability to take charge of one's own learning“versteht Holec die Fähigkeit des Lerners, sein eigenes Lernen zu steuern. Dabei setzt er selbstgesteuertes 
Lernen mit autonomem Lernen gleich. Der autonome Lerner trägt somit zunehmend die Verantwortung bezüglich der Ziele, Inhalte, Lerntechniken und Evaluation seines Lernprozesses (vgl. Chan 2000: 57f). Diese Fähigkeit ist nach Holec (vgl. 1979: 3f) jedoch nicht angeboren, sondern muss entweder auf einem natürlichen Weg oder, wie es meistens der Fall ist, erst durch gesteuertes Lernen erworben werden. Auch Little (1991: 5ff) betrachtet die Befähigung des Lerners zur Eigenverantwortung als unbedingt notwendig.

Die Annäherung an autonomes Lernen wird durch viele Faktoren begünstigt. Ein verändertes Rollenverhalten auf Lehrerseite bildet eine grundlegende Voraussetzung, wodurch die Entwicklung von autonomem Lernen erst beginnen kann. Subjektive Vorstellungen vom Fremdsprachenlernen, individuell bevorzugte Lernstile und Lernstrategien gilt es den Lernenden dabei bewusst zu machen, damit eine Bereitschaft zur Annahme neuer Lernwege entstehen kann. Dadurch kann der Lerner zunehmend Verantwortung übernehmen und das Lernen verstärkt den eigenen Bedürfnissen anpassen. Veränderte Rollen und veränderte Ziele können sich jedoch schwerlich mit Material verwirklichen, das fremdbestimmte Ziele und Schritte impliziert. Lerner sollen deshalb vermehrt selbst gewähltes Material in ihren Lernprozess mit einbringen, welches ihnen durch Authentizität individuelle Lernzugänge ermöglicht (vgl. Wettemann 2004, besonders Kap. 2: Die Weichenstellung für ein lebenslanges Lernen).

\subsection{Theoretische Grundlagen}

Aus der Perspektive der kognitiven Lerntheorie sind Wahrnehmen und Verstehen konstruktive Operationen, die jeder Mensch individuell und auf der Grundlage seines spezifischen Welt- und Erfahrungswissens durchführt. „Lernen wird in dieser Theorie als eine aktive Tätigkeit verstanden, die der Lerner unabhängig durchführt und die nicht von außen beeinflusst oder kontrolliert werden kann" (Wolff 1999: 43). Die konstruktivistische Lerntheorie führt diesen Erklärungsversuch noch einen Schritt weiter. Sie geht nämlich davon aus, dass das menschliche Gehirn ein in sich geschlossenes System darstellt und sich selbst organisiert. Laut Wolff (vgl. 1994: 415) ist Lernen folglich als Konstruktion von Wissen ein Produkt dieser Selbstorganisation und unterliegt deshalb der Verantwortung des Individuums. Unter dieser veränderten Perspektive stellt sich Lernen als individueller und vom Lerner eigenständig gesteuerter Lernprozess heraus, bei dem sich jeder Lerner sowohl bezüglich Lernweg als auch Ergebnis unterscheidet. Deshalb stimme ich mit Wolff (ibid. 427) darin überein, dass die kognitive und die konstruktivistische Lerntheorie die theoretischen Grundlagen des autonomen Lernens bilden und möchte deshalb die Zusammenhänge mit meinem dramapädagogischen Ansatz weiter darstellen.

\subsection{Lernerautonomie und dramapädagogischer Ansatz}

Beim autonomiefördernden Fremdsprachenunterricht werden die Lerner durch die Bewusstmachung ihrer individuellen Lernprozesse dazu angeregt, eine 
aktive Rolle beim Lernen einzunehmen. Dabei versuche ich stufenweise, die Lerner zu einer wachsenden Selbststeuerung zu befähigen, die sich in Veränderungsprozessen ihres Denkens und Handelns widerspiegelt.

In der Übertragung auf meinen dramapädagogischen Ansatz für den Anfängerunterricht werden die tragenden Lernprinzipien aus diesem Denkmodell umgesetzt. Zur Verdeutlichung möchte ich die wichtigsten kurz nennen:

- Die Förderung authentischer Interaktion in der Zielsprache entspricht dem Prinzip der authentischen Lernumgebung, in der Lerninhalte konkret gebraucht werden.

- Die bewusste Fokussierung auf Verarbeitungs- und Lernprozesse bezieht sich auf das Prinzip der Wissensverarbeitung.

- Die Sozialform der Gruppenarbeit unterstreicht das Prinzip des kooperativen Lernens. Im Austausch des individuellen Wissens bringen Lerner ihre subjektiven Welten zusammen und bauen dadurch eine neue Welt auf, in der sich alle orientieren können (vgl. Wolff 1994: 427, 1999: 44).

Als Konsequenz sehe ich in Übereinstimmung mit Wolff (ibid., Little 1991: $15 f f$ u.a.) eine Unterrichtspraxis, die dem Lerner die Möglichkeit zum selbstbestimmten, autonomen Lernen eröffnet, als unverzichtbar an. Die Planung muss folglich für alle transparent und flexibel werden, d.h. die Lernziele und deren Begründungen müssen offen gelegt und ihre Aneignungsprozesse variabel werden. Mein Ziel ist es deshalb, den dramapädagogischen Ansatz zu erweitern und ihn mit dem Ansatz des autonomen Lernens zu verbinden, damit die Lerner dazu befähigt werden, die Verantwortung für ihren eigenen Lernprozess zunehmend übernehmen zu können. In der Modelleinheit wird dies durch die Bewusstmachung über die eigenen und das Ausprobieren neuer Lernwege begünstigt. Weiterhin dienen die video-dokumentierten Lernerergebnisse zur Reflexion über den individuellen Lernzuwachs.

\section{Erweiterung des dramapädagogischen Ansatzes}

Auf dem Kontinuum von Kao/O’Neill (1998: 5ff) werden die dramapädagogischen Unterrichtsformen ${ }^{9}$ "from totally controlled language exercises and scripted role-plays through the semi-controlled approach of the scenario, to the kind of open communication of process drama" eingeteilt. In ihren Erläuterungen stellen sie jedoch heraus, dass diese drei Inszenierungsformen ${ }^{10}$ entsprechend des Sprachstandes der Lerner eingesetzt werden sollen, was

\footnotetext{
9 Die von Kao/O'Neill verwendete Bezeichnung Process Drama entspricht dem von Schewe (1993) verwendeten Begriff Drama in Education, denn ,in Britain the terms ... 'drama in education' are almost synonymous with process drama” (vgl. Kao/O’Neill 1998: 12).

10 Weiterhin ist die Formulierung „Inszenierungsformen“ meine Übersetzung von Kao/O’Neills „drama approaches“. Für eine genauere Unterscheidung zwischen Inszenierungsmuster, -formen und gestaltete Improvisation siehe Schewe 1993: 282ff.
} 
meiner Meinung nach einem Kontinuum widersprechen würde. Dies betont auch Schewe, der zwar die ganz offene Inszenierungsform als Kern eines dramapädagogischen Fremdsprachenunterrichts sieht, in die jedoch Elemente von anderen, eher geschlossenen Inszenierungsformen integriert werden können (vgl. 1993: 282). Insbesondere für L2-Anfänger sind geschlossene Übungsformen als erster Schritt unverzichtbar, da sie dem Lerner Raum geben, sich sowohl auf formale Korrektheit als auch auf Aussprache, Intonation und Flüssigkeit konzentrieren zu können. Erst wenn diese sprachlichen Mittel integriert und automatisiert sind, kann zu halboffenen Übungsformen übergegangen werden. Wie sollten also geschlossene Übungsformen aussehen, damit Lerner die kommunikativen Ziele rasch in einer halboffenen Übungsform, einem so genannten Szenario, anwenden können?

Den mündlichen Übungen sollten Schritte des Verstehens und der Analyse vorausgehen (vgl. Gerngroß/Krenn/Puchta 1999), wofür ich folgende Schritte vorschlage: Die Lerner bekommen Minidialoge, in denen die kommunikativen Ziele durch eine Text-Bildkombination ${ }^{11}$ veranschaulicht werden. Daran schließen sich zur Bewusstmachung des Klangbildes der neuen Sprache entsprechendeHörübungen an. Dannwerden von den Lernern dieerforderlichen Redemittel entsprechend der kommunikativen Ziele ${ }^{12}$ zusammengetragen. Nach diesem ersten Kontakt, wobei der Schwerpunkt auf das Verstehen gerichtet ist, folgt nun ein Analyseschritt, wo z.B. (siehe Anhang B: 1. Dramapädagogische Einheit) die Personalpronomen und entsprechenden Konjugationsendungen visuell als Enhanced Input hervorgehoben (vgl. Ellis 2005: 13) und benannt werden: Personalpronomen, Konjugation. Weiterhin wird die Intonation durch Pfeile veranschaulicht. Darauf folgen geschlossene Übungen, die unverzichtbare sprachliche Vorübungen für die Szenarien darstellen.

\subsection{Geschlossene Übungsformen mit Kärtchen}

Das Arbeiten mit Kärtchen ${ }^{13}$ dient in dieser Modelleinheit als zentrale Übungsform zur Integration und Automatisierung von Redemitteln u.a. Wie damit kreativ und lernerzentriert gearbeitet werden kann, möchte ich an einigen Varianten genauer erklären: Auf der Vorderseite jedes Kärtchens soll der Satz

\footnotetext{
${ }^{11}$ Geeignete Beispiele finden sich zuhauf in modernen, kommunikativ orientierten Lehrwerken.

12 Aus einer Studie (Wettemann 2004: 54), die sich auf über 100 befragte Lerner in Mexiko unterschiedlichster Niveaustufen stützt, hat sich gezeigt, „dass im Unterricht selbst kaum Ansätze für autonomes Lernen zu finden sind, was indirekt auch auf den mangelnden Wissensstand der Lehrer darüber schließen lässt.“ Als Wendepunkt zum traditionellen expliziten Grammatikwissen und Vokabeln lernen bildet dafür die Bewusstmachung von kommunikativen Zielen einen ersten unverzichtbaren Schritt.

13 Um Bereitschaft für neue Lernformen zu fördern (vgl. Weskamp 2001: 41), wurde das Arbeiten mit Kärtchen im Vorab besprochen. So konnten die Lerner Erfahrungen aus ihrer eigenen Anwendung zum Ausdruck bringen. Dann wurden „Negativbeispiele“ von beschriebenen Kärtchen aus dem Plenum mit „Modellkärtchen“ verglichen und somit veranschaulicht, WAS darauf geschrieben wird, WIE es notiert wird und WIE damit geübt werden kann (language learning awareness).
} 
auf Deutsch mit den entsprechenden Markierungen notiert werden und auf der Rückseite die pragmatisch angemessene Übertragung in die Muttersprache. ${ }^{14}$ Die zuvor erarbeiteten und auf Kärtchen geschriebenen Redemittel, Chunks, Strukturen etc. können jetzt vielseitig automatisiert und integriert werden. Dabei sollte den Lernern bewusst und erfahrbar gemacht werden, dass rezeptives Sprachkönnen ${ }^{15}$ (Verstehen) nicht mit produktivem Sprachkönnen (Sprechen) gleichzusetzen ist. ${ }^{16}$ In Partnerarbeit sollte folgendermaßen erst rezeptiv und dann produktiv geübt werden.

- Partner A liest deutsch vor und kontrolliert B's Verstehen. Partner B hat keine Kärtchen, konzentriert sich auf das Verstehen und gibt A die pragmatisch richtige Übersetzung. Diese Übungsform ist für B rezeptiv in Bezug auf die Fremdsprache.

- Partner A liest spanisch vor und kontrolliert B's Verstehen. Partner B hat keine Kärtchen und versucht die pragmatisch richtige Übersetzung zu produzieren. Diese Übungsform ist für B produktiv in Bezug auf die Fremdsprache.

Eine weitere Anwendung besteht z.B. in Minidialogen ${ }^{17}$, die auf Vorder- und Rückseite aufgeteilt sind. Das Verstehen wird durch den Kontext unterstützt, da auf den Kärtchen ohne Übersetzungshilfe gearbeitet wird. Geübt wird ebenfalls in Partnerarbeit: In der ersten Runde hat z.B. A die Vorderseite aller Kärtchen vor sich liegen und B die Rückseite. Diese Übung kann dann mit Read and Look up (s.u.) kombiniert werden. Zur weiteren Automatisierung hat dann nur noch A Kärtchen und B muss die Antworten rekonstruieren etc. Im Mittelpunkt stehen Zuhören und Konzentration auf den Inhalt.

In beiden Übungsformen übernehmen die Lerner gegenseitige Verantwortung für ihren aktiven Lernprozess und sind durch Lerntransparenz und Eigenregulierung in der Regel stärker motiviert. Der Lehrer fungiert hierbei als Helfer, um Flüssigkeit, Intonation und Aussprache in jeder Gruppe zu erreichen (vgl. Kap. 3.3).

\subsection{Geschlossene Übungsformen mit Information Gap: Read and Look Up}

Wie kann der dramapädagogische Ansatz dazu beitragen, kommunikative Kompetenz aufzubauen und freieres Sprechen zu fördern? Durch den kreativen Umgang mit dieser ebenfalls noch geschlossenen Übungsform möchte ich aufzeigen, wie Sprache weiter integriert und automatisiert werden kann. Dazu

\footnotetext{
14 Besonders anschaulich zeigt es sich an den „Chunks für unterrichtliche Kommunikation“ (siehe Anhang A: Tabelle 1).

15 Dieser Punkt begründet sich durch die Output-Hypothese.

16 Wenn Lerner außerdem zu schnell zur (freien) Produktion aufgefordert werden, führt dies meiner Erfahrung nach oft zu einer Überforderung und auch zu Lernfrustration.

17 Sehr bildlich wird das an den Minidialogen aus Anhang A: Tabelle 2.
} 
verwende ich die Übungsform information gap, die normalerweise nur im rezeptiven Lesebereich als Verstehensübung verwendet wird. Im ersten Schritt sollen sich die Lerner auf den Inhalt konzentrieren und die Satzteile bzw. die Dialogstücke (siehe Anhang A: Tabelle 2) miteinander verbinden. Wie könnte diese Übungsform noch verwendet werden, um Aussprache, Intonation, Flüssigkeit und Korrektheit zu üben?

Dazu arbeiten die Lerner erneut mit einem Partner. Jeder faltet sein Arbeitsblatt der Länge nach so, damit er nur einen Teil des Dialoges sehen kann. Lerner A beginnt mit der Methode read, repeat silently and look $u p^{18}$, wobei im freien Sprechen (look up) auf Augenkontakt zu achten ist. Lerner B konzentriert sich auf das Verstehen und wählt dann die entsprechende Antwort aus, vollzieht jedoch ebenfalls die drei Schritte usw. ${ }^{19}$ Nach der ersten Phase hat z.B. nur noch A das Blatt und beginnt mit den Minidialogen, Lerner B antwortet ohne Text mit den zuvor praktizierten Redemitteln. A kontrolliert und kann Hilfestellungen geben etc. Dann wird getauscht (vgl. Kap. 2.2: pushed output). Die Aufgabe des Kursleiters ist während der Arbeitsphase wie zuvor.

In der folgenden Übungsphase findet eine Inszenierung des Dialogs statt. Zwei Lernergruppen stehen sich gegenüber. Der Schwierigkeitsgrad wird vom Lehrer zunehmend erhöht, bis der Moderator den Gruppen nur noch Stichworte gibt (siehe die unterstrichenen Wörter im Anhang A: Tabelle 2). Während Gruppe A den Satz rekonstruiert und sich für ein flüssiges Sprechen im Chor vorbereitet, hat Gruppe B Zeit die Antwort zu rekonstruieren usw. Diese Übungsform ist variabel, in dem z.B. Lerner die Moderator-Rolle übernehmen, die Großgruppe weiter aufgeteilt wird oder die Inszenierung bis zu einem Duellkampf ${ }^{20}$ gesteigert wird.

Diese Formen von geschlossenen Übungen unterscheiden sich grundsätzlich von denen, die Kao/O'Neill (1998: 5ff) aufführen und meiner Meinung nach nicht den Bedürfnissen erwachsener Lerner entsprechen. ${ }^{21}$ Ferner stehe ich konträr zu Kao/O'Neills Behauptung, dass “the pre-determined features of these activities restrict learners from progressing to higher levels in using the target language“ (1998: 9). Ganz im Gegenteil können Formen von geschlossenen

\footnotetext{
18 Für die Methode read and look up wurde ich von Kakuschky (2004: 41ff) inspiriert. Weiterhin habe ich read and look up um die Zwischenstufe repeat silently erweitert, um die „rhythmisch-klangliche Basis“ zu integrieren (vgl. Campadieu 1999:159).

${ }^{19}$ Am einfachsten ist es, wenn es der Lehrer mit einem Kursteilnehmer einmal vor der Gruppe vormacht, anstelle es abstrakt zu erklären.

${ }^{20}$ Dabei konnte ich an den Lippenbewegungen der beobachtenden Lerner ihre aktive Beteiligung ablesen, wie sie ebenfalls versuchten die Sätze zu rekonstruieren. Weiterhin habe ich diese Form von Information gap auch schon in einer internen Lehrerfortbildung in Mexiko mit über 30 Teilnehmern angewendet (mit englischen Redemitteln) und darf auch hier von einer besonders hohen Spannungsgeladenheit - im Sinne einer dramapädagogischen Inszenierung sowohl auf Teilnehmer- als auch auf Beobachterseite berichten.

${ }^{21}$ Kao/O’Neill zählen hierzu Sprachspiele, Rollenspiele und Simulationen auf. Als Beispiel für Sprachspiele geben sie „The seat on my left is free“ an, das für erwachsene Lerner peinlich sein kann und deshalb unangemessen ist. Unter Rollenspielen verstehen sie das Einüben und Nachspielen von vorgefertigten Dialogen etc. Als weitere Kritik an der Übungsform Rollenspiel im Fremdsprachenunterricht siehe Schewe 1993: 154, insbesondere Kapitel IV: Spielen als Farce?
} 
Übungen für L2-Anfänger den Rahmen bilden, innerhalb dessen sie sich verstärkt auf formale Korrektheit, Flüssigkeit, Intonation und Aussprache konzentrieren können. Bei der Einführung neuer Strukturen schreibt Even (2003: 158) dieser Übungsform auch bei fortgeschrittenen Lernern einen hohen Nutzen zu.

\subsection{Videounterstützter Pendelgang zu halboffenen Übungsformen}

Szenarien bilden die Grundlage für halboffene Übungsformen, die Kao/O'Neill (1998: 9) mit Di Pietros (1987) Strategic Interaction gleichsetzen, wobei sie den Schwerpunkt "on reaching a communicative goal within the shelter of the group" legen (Kao/O'Neill 1998: 10). Dem sozialen Lernen wird dabei eine tragende Rolle zugestanden: Indem sich die Lerner verstärkt einbringen können, sind sie auch zunehmend motiviert, denn „[s] tudents care more about a situation and the information it contains, and understand it better when they have worked to make its meaning" (ibid. 10). Alle Unterrichtseinheiten verlaufen dabei nach den Phasen der Probe, Aufführung und Nachbesprechung. Unberücksichtigt bleibt bei Kao/O'Neill jedoch, dass bei Sprachanfängern nicht bei der Probephase begonnen werden kann, sondern die Einheiten der Analyse, Integration und Automatisierung vorangestellt werden müssen, die ich zuvor dargestellt habe. Weiterhin zeichne ich im Unterschied zu Kao/O'Neill nur die Umsetzungsphasen auf Video auf und verwende diese vielseitig für weitere Arbeitsprozesse.

Zur Veranschaulichung möchte ich exemplarisch die sechs dramapädagogischen Umsetzungen ${ }^{22}$ der ersten zwei Wochen meines Intensivkurses darstellen: Schon in meiner ersten dramapädagogischen Umsetzung stehen kommunikative Ziele im Mittelpunkt. Durch Inszenierung wird den Teilnehmern die Bedeutung der Ziele erfahrbar gemacht. In der zweiten werden diese Ziele erweitert und die Lerner können sich schon in der Erarbeitungsphase zunehmend durch eigene Vorschläge einbringen. Danach erhalten die Beobachter zusätzliche sprachliche Mittel und Kriterien, um in den Handlungsprozess eingreifen zu können. In der dritten Umsetzung wird die Ausgestaltung und das Handeln in den Rollen ${ }^{23}$ aufgebaut, das vielseitige Vorteile bietet: Im Schutz der Rolle fühlen sich die Lerner freier und haben beispielsweise weniger Angst, beim Sprechen Fehler zu machen oder neue verbale und nonverbale Handlungsalternativen auszuprobieren. Die Rollenattribute beginnen mit Herkunftsland, Wohnort und Sprachen, werden jedoch in jeder neuen Einheit erweitert. Der Rollenaufbau wird in der Probephase mit dem zuvor praktizierten Dialogmuster kombiniert. Diese Umsetzung wird jetzt auf Video aufgenommen. Da sich alle das erste Mal „deutsch sprechen sehen“, sollte dem Lachen über sich selbst Vorzug vor der geplanten Reflexion gegeben werden.

\footnotetext{
22 Zum besseren Verständnis empfehle ich parallel zu den kurzen Erläuterungen der dramapädagogischen Umsetzungen die entsprechendenden Übersichtstabellen anzuschauen: Anhang B: 1-6 Dramapädagogische Einheit.

${ }^{23}$ De Pietro betont, dass das Arbeiten mit Rollen sehr motivierend für das Lernen ist (zitiert in Kao/O’Neill 1998: 10).
} 
In der vierten Umsetzung werden nun Handlung und Rolle in ein Szenario eingebettet. Durch einen minimalen Kontext kann eine „als ob" Situation geschaffen werden, wodurch eine Basis für Interaktion vorhanden ist, in der Grund, Absicht und Ziel der Kommunikation im Hier-und-Jetzt erfahrbar wird (vgl. Ortner 1998: 148). Durch die wachsende Authentizität und den Schutz der Rolle steigt die Bereitschaft bei den Lernern neue Handlungsmuster und -alternativen auszuprobieren. In der videounterstützten Nachbesprechung werden dann Fehler reflektiert und Verbesserungsvorschläge eingebracht.

In der Folgeeinheit wird das Szenario erweitert. Die Rahmensetzung bildet eine Situation in der Cafeteria/Uni Berlin. Alle kommen aus unterschiedlichen Ländern, sind im ersten Semester und möchten neue Leute kennen lernen. Durch die folgende Inszenierung wird ein Sprechen in unvorhersehbaren Situationen geschaffen. Die Umsetzungen bauen kontinuierlich auf die vorhergehenden auf. Dies bildet in meinem Ansatz ein tragendes Prinzip für die dramapädagogische Umsetzungim Anfängerunterricht: DieEinheiten dürfen dahernicht unabhängig voneinander entwickelt werden, sondern müssen, wie bei einem Zwiebelmodell, aufeinander aufbauen und die vorhergehenden Stufen immer mit einschließen. In dieser Umsetzung werden jetzt die kommunikativen Ziele um „fragen können, wie es jemanden geht“ und „sagen können, was man trinken möchte" erweitert. Nachdem die sprachlichen Mittel in der vorhergehenden Übungsphase integriert und automatisiert wurden, werden die Lerner in die Situation und Handlungsintention eingeführt. Für die dramapädagogische Umsetzung werden in Partnerarbeit neue Rollen (Name, Land, Stadt, Alter etc.) entwickelt, die den anderen Gruppen jedoch nicht gezeigt werden dürfen. Dadurch wird Spannung in ihrer ursprünglichen Bedeutung ${ }^{24}$ geschaffen: Wer wird mit wem spielen und welche Rolle wird der Andere haben? Dafür würfelt der Moderator die Akteure neu zusammen, gibt jedoch erst vor jedem „Auftritt“ bekannt, wer mit wem spielt. Beispielsweise spielen Aranza und Uriel in einer Szene. Während Uriel sich den Beobachtern in seiner Rolle ${ }^{25}$ vorstellt, wartet Aranza, damit sie nichts über die andere Rolle mitbekommt, vor der Tür. Dann geht Uriel raus, Aranza kommt, stellt sich in ihrer Rolle vor, setzt sich an den Tisch, ruft Uriel und die Szene beginnt.

Da es bei solcher Strategic Interaction im Vergleich zu geschlossenen Formen $\mathrm{zu}$ unerwarteten Herausforderungen kommen kann, erwächst zusätzlich Spannung aus der Gruppendynamik. Bei schwächeren Lernern sollte deshalb nach Wunsch auch mit einem Doubling gearbeitet werden, wobei sich ein anderer Lerner hinter den Akteur stellt und ihn bei „Ausfällen“ wie ein Souffleur unterstützt. Durch dieses soziale Lernen (Wolff 1999) wird dem Lerner eine wachsende Selbstbestimmung ermöglicht, die in der folgenden Arbeitsform weiter fortgesetzt wird.

\footnotetext{
${ }^{25}$ Zur Veranschaulichung möchte ich Uriels Rollenpräsentation exemplarisch vorstellen: Er kommt herein, baut Blickkontakt mit den Beobachtern auf und beginnt sich vorzustellen: „Guten Tag. Ich heiße André Shevchenko. Ich komme aus Russland. Ich wohne eigentlich in Belgrad, ich bin 23 und studiere Deutsch in Berlin. Ich bin in der Cafeteria und möchte neue Leute kennen lernen. Ich bin super motiviert.“
} 
Bevor das Video zur Reflexion herangezogen wird, sollen sich schwächere Lerner so genannte Regisseure als Helfer aus der Großgruppe auswählen. Dann werden die Videosequenzen unter den schon bekannten Kriterien sowie „falsche/unpassende Interaktion; fehlender Satz oder Reaktion“ etc. analysiert, damit Lerner ihre Stärken und Schwächen erkennen können: ein tragendes Prinzip der Lernerautonomie. Auf der Grundlage dieser Reflexion wird die Szene vom Regisseur in den Kleingruppen erneut eingeübt und dann für die nächste Umsetzung um die Rolle eines Kellners erweitert. Die Hilfestellung untereinander wird meiner Erfahrung nach sehr positiv angenommen und fördert das Gruppengefühl.

Diese sechs dramapädagogischen Umsetzungen bildeten den Inhalt der ersten zwei Wochen des Intensivkurses. Um den Rahmen dieses Artikels nicht zu sprengen, deute ich den Verlauf der dritten Woche nur noch an. Die kommunikativen Ziele der letzten Woche waren „auf dem Flohmarkt etwas kaufen können“ und „über den Preis verhandeln können“. Unter den grammatischen Zielen stand die Entwicklung eines Bewusstseins über internationale Wörter, Substantive und deren grammatisches Geschlecht im Vordergrund. Zum Abschluss wurde der Sprachlernprozess der drei Wochen mit Hilfe der Videoaufzeichnungen noch einmal veranschaulicht.

\section{Resümee und Blick nach vorne}

\subsection{Erfahrungen aus dem Intensivkurs}

Auf der Grundlage der oben beschriebenen dramapädagogischen Unterrichtseinheiten führte ich im Sommer 2006 in Puebla (Mexiko) im Centro de Idiomas einen Intensivkurs für Sprachanfänger ohne Vorkenntnisse durch. Der Kurs erstreckte sich über drei Wochen, wobei die 4-stündigen Unterrichtsblöcke von Montag bis Freitag stattfanden. Die elf mexikanischen Teilnehmer waren im Durchschnitt 18 Jahre alt. Am letzen Unterrichtstag erhielten alle Teilnehmer zur Erweiterung der videounterstützten Auswertung einen Evaluationsbogen (siehe Anhang A). Dieser enthielt Fragen sowohl zur allgemeinen Reflexion über den Unterricht als auch zu den autonomiefördernden und dramapädagogischen Ansätzen. Da beide Ansätze sehr stark ineinander greifen, habe ich in der Auswertung nicht mehr versucht zwischen diesen einen klaren analytischen Trennstich zu ziehen.

Die Arbeitsformen mit Kärtchen, read, repeat silently and look up u.a. schienen den Teilnehmern nicht aufgedrängt, sondern sehr motivierend und machten ihnen erfahrbar, dass die Zielsetzungen dadurch schnell erreicht werden konnten. Sehr motivierend fanden sie auch die Einbettung der Dialoge in zunehmend komplexere Szenarien, in denen sich alle kreativ und entsprechend ihrer Bedürfnisse einbringen konnten. Darin konnte das zuvor Erarbeitete jetzt im Kontext angewendet und dadurch besser behalten werden. Durch den Rollenschutz bauten die Lerner schnell ihre anfänglichen Ängste ab und wurden zunehmend bereit auch neue verbale und nonverbale Handlungsalternativen 
auszuprobieren. ${ }^{26}$.

Besonders die Arbeit mit den Videos wurde als positiv und motivierend bezeichnet, wobei folgende Punkte im Evaluationsbogen häufig genannt wurden: Die Arbeit mit den Videos

- „war eine starke Herausforderung für mich und hat mich dazu gezwungen, das Gelernte auch wirklich anwenden zu können“,

- „hat es mir ermöglicht, meine Fehler selbst zu erkennen und zu verbessern“

- „ist eine sehr vielseitige Art und Weise zu lernen“ und

- „hat eine starke Gruppenbildung gefördert“.

Das vielseitige und abwechslungsreiche Lernen wurde auch mit anderen fremdsprachlichen Unterrichtserfahrungen verglichen, in denen das Lernen als passiv und schwierig empfunden wurde: „Ich dachte deshalb, dass es sehr schwierig ist, habe aber das Gegenteil erfahren.“ oder „Es schien sehr leicht zu gehen und ich habe in drei Wochen extrem viel und schnell gelernt." Diese von allen als positiv empfundene Unterrichtsgestaltung wird von einem anderen Lerner so beschrieben: „Dies ist meine zweite Fremdsprache, die ich lerne, aber es ist die beste Form gewesen, in der ich bis jetzt gelernt habe." Und ein anderer drückt seine Begeisterung durch seinen flow effect aus: „Der Kurs hat mich super motiviert, denn es gab Momente in denen ich mein Spanisch vergaß und Deutsch einfach super fand." Weitere Kommentare hoben die holistische Perspektive des Lernens heraus: „Ganz im Ernst, mir hat der Kurs super gefallen, weil wir wirklich alles ${ }^{27}$ gelernt haben!“ Abschließen möchte ich die Auswertung mit den Kommentaren der Teilnehmer auf den Satzanfang: Wenn der Unterricht immer so wäre: ${ }^{28}$

- „hätten wir mehr Freu(n)de“

- „würden wir mehr lernen“

- „könnten wir flüssiger sprechen“

- „würden wir schneller lernen“.

Die Ergebnisse des Evaluationsbogens bestätigen durchgehend die vorteilhafte Verbindung des autonomiefördernden und dramapädagogischen Ansatzes. Das zeigt sich in der starken Motivationsförderung, da sich alle in den Folgesprachkurs eingeschrieben haben.

\footnotetext{
26 Dieses Ausprobieren und Hypothesen-Überprüfen wird so genannten „good learners“ zugesprochen (vgl. Rubin 1975; Stern 1975).

27 Durch die Formulierung „weil wir wirklich alles gelernt haben“ möchte der Lerner zum Ausdruck bringen, dass er sich durch die ganzheitlichen Lernsituationen ein konkretes Bild über Sprache, Land, Kultur und deren Menschen etc. machen konnte und dass ebenfalls das „Lernen lernen“ ihm sehr geholfen hat.

28 Diese offene und einladende Fragestellung habe ich von Even (2003) übernommen.
} 


\subsection{Synthese}

Ich versuchte darzustellen, dass der dramapädagogische Ansatz sich nicht auf die Anwendung in der Interaktion begrenzen muss (vgl. Ortner 1998: 148), sondern die Schritte der Analyse, Integration und Automatisierung schon von der ersten Unterrichtstunde an kreativ und motivierend mit einschließen kann. Für die Lernzieldefinition habe ich mich an den globalen Kann-Beschreibungen orientiert, wo vom Lerner am Ende der Niveaustufe A1 kein „freies Sprechen in unvorhersehbaren Situationen“ erwartet wird, sondern „mit wenigen, einfachen und auswendig gelernten Ausdrücken und Sätzen vertraute Situationen bewältigen zu können, die ganz alltägliche und konkrete Bedürfnisse betreffen" (Profile Deutsch 2002: 75). Diese Fertigkeiten werden durch das autonomiefördernde Lernen vorbereitet und in geschlossenen Übungsformen automatisiert. Durch diese Verfahren rücken die Subjektivität und Individualität der Lerner in den Mittelpunkt. Die Lerner erfahren eine neue Abfolge von Unterricht, die ihre Bedürfnisse ${ }^{29}$ und Lernschwierigkeiten mit einschließt. Die Anwendungen in den halboffenen Übungsformen bauen langsam auf einander auf und werden durch fiktive Rollen und Szenarien immer authentischer, wobei durch Doubling, Soufflieren und Regieübernahme zunehmende Selbstgestaltung ermöglicht wird. In den dramapädagogischen Umsetzungen wird die Bedeutung des Gelernten für alle im „Hier-und-Jetzt“ (Ortner 1998: 148) erfahrbar. Dies bildet nach Little (1991:4) die Voraussetzung für ein autonomes Lernen, durch das die Barrieren zwischen Leben und Lernen überwunden werden. Zusätzlich wird auf der Grundlage einer kontinuierlichen Videoaufzeichnung und -reflexion der Sprachlernprozess in den Mittelpunkt gerückt, der klar dokumentiert ist und vom Lerner selbst evaluiert werden kann.

Durch die Einbeziehung von Formen autonomen Lernens kann der dramapädagogische Ansatz also schon im Anfängerunterricht ansetzen. Die Zielsetzung formeller Korrektheit und Flüssigkeit bilden dabei keinen Widerspruch zur Lernerorientierung. Wie sich aus der Auswertung des Intensivkurses ergeben hat, führt die Kombination beider Ansätze zu einem schnellen Spracherfolg und zur Aufrechterhaltung der Motivation. Deshalb plädiere ich, insbesondere bei Sprachlernanfängern, für die Verschmelzung von autonomieförderndem und dramapädagogischem Fremdsprachenunterricht.

\section{Bibliographie}

Bausch, Karl-Richard / Christ, Herbert / Krumm, Hans-Jürgen (eds.) (2003): Handbuch Fremdsprachenunterricht. Tübingen / Basel: Francke

\footnotetext{
29 Jede Gruppe brauchte für ihre Szene zusätzliche komplexe Chunks (Perfekt-, Imperfektoder Imperativformen), die bereitgestellt und unanalysiert integriert wurden. Davon möchte ich einige hervorheben: Gib mir alles, was du hast!/Kann ich dir helfen?/Er hat mich verarscht./Von mir wollte er nur 15 Euro./Verschwinde./Vergiss den Ventilator nicht./Dann reicht das Geld nicht mehr für das Handy./Der CD-Player kommt aus China./Wie viel hast du bezahlt?/Was hast du gekauft? etc. Die von den Lernern benötigten komplexen Chunks spiegeln das autonomiefördernde Lernen wider (vgl. Wolff 1999: 44).
} 
Bolton, Gavin (1993): A Brief History of Classroom Drama: British and Other English-speaking Influences. In: Schewe, Manfred / Shaw, Peter (eds.): Towards Drama as a Method in the Foreign Classroom. Frankfurt/M.: Peter Lang, 25-42

Campadieu, Petra (1999): Warum lernen manche Menschen leicht und andere nur mit Mühe eine Fremdsprache? In: Deutsch als Fremdsprache. Zeitschrift für Theorie und Praxis des Deutschunterrichts für Ausländer 3, 156-159

Chan, Wai Meng (2000): Metakognition und der DaF-Unterricht für asiatische Lerner. Möglichkeiten und Grenzen. München: Waxmann

Düwell, Henning (2003): Personale Aspekte beim Lehren und Lernen fremder Sprachen. In: Bausch et al: Handbuch Fremdsprachenunterricht. Tübingen / Basel: Francke, 347-352

Edelhoff, Christoph / Weskamp, Ralf (eds.) (1999): Autonomes Fremdsprachenlernen. Ismaning: Hueber

Edmondson, Willis / House, Juliane (2000): Einführung in die Sprachlehrforschung. Tübingen: Francke

Ellis, Rod (2005): Instructed Second Language Acquisition. A Literature Review. Wellington: Wãhanga Mahi Rangahau, Research Division

Even, Susanne (2003): Dramagrammatik. Dramapädagogische Ansätze für den Grammatikunterricht Deutsch als Fremdsprache. München: iudicium

Gass, Susan M. / Madden, Carolyn (eds.) (1985): Input in Second Language

Acquisition. Rowley, Mass.: Newbury House

Gerngroß, Günter / Krenn, Wilfried / Puchta, Herbert (1999): Grammatik kreativ. Materialien für einen lernerzentrierten Grammatikunterricht. Berlin: Langenscheidt

Gibbons, Kara (2005): Towards Process Drama in the German Classroom at Primary Level in Ireland. An Investigation into the Suitability of the Dramatic Method and in particular, Process drama, in the Teaching of German to Younger Learners in Ireland. MPhil Thesis in German at National University of Ireland, Cork

Glaboniat, Manuela / Müller, Martin / Schmitz, Helen / Rusch, Paul / Wertenschlag, Lukas (2002): Profile Deutsch. Berlin: Langenscheidt

Gnutzmann, Claus (2003): Language Awareness, Sprachbewusstheit, Sprachbewusstsein. In: Bausch et al: Handbuch Fremdsprachenunterricht. Tübingen / Basel: Francke, 335-339

Henrici, Gert (1995): Spacherwerb durch Interaktion? Eine Einführung in die fremdsprachenerwerbsspezifische Diskursanalyse. Baltmannsweiler: Schneider

Holec, Henri (1979): Autonomy and Foreign Language Learning. Strasbourg: Council of Europe

Hufeisen, Britta; Neuner, Gert (2004): Tertiärsprachendidaktik. Fernstudienangebot Germanistik Deutsch als Fremdsprache. Berlin: Langenscheidt 
Kakuschky, Gerhard (2004): Gedichte lernen - aber ja!!! In: DaF Brücke 6, 41-43

Kao, Shin-Mei; O'Neill, Cecily (1998): Words into Worlds. Learning a Second Language through Process Drama. Stamford et al.: Ablex

Krashen, Stephen (1982): Second Language Acquisition and Second Language Learning. Oxford: Pergamon

Lemcke, Christiane / Rohrmann, Lutz / Scherling, Theo (2002): Berliner Platz 1. Deutsch im Alltag für Erwachsene (Lehrbuch). Berlin: Langenscheidt

Little, David (1991): Learner Autonomy. Definitions, Issues and Problems. Dublin: Authentik

Long, Michael (1983): Native Speaker/Non Native Speaker Conversation in the Second Language Classroom. TESOL 82, 207-225

O'Brien, Kathleen (2004): Teaching Beginners French to an Irish Primary Fourth Class. MA Thesis in Drama and Theatre Studies, National University of Ireland, Cork

Ortner, Brigitte (1998): Alternative Methoden im Fremdsprachenunterricht. Lerntheoretischer Hintergrund und praktische Umsetzung. Ismaning: Hueber

di Pietro, Robert J. (1987): Strategic Interaction. Learning Languages through Scenarios. London: Cambridge University Press

Rubin, Joan (1975): What the ,Good Language Learner' Can Teach Us. In: TESOL Quarterly 1, 41-51

Schewe, Manfred (1993): Fremdsprache inszenieren. Zur Fundierung einer dramapädagogischen Lehr- und Lernpraxis. Oldenburg: Zentrum für pädagogische Berufspraxis, Carl von Ossietzky Universität Oldenburg

Schewe, Manfred (2000): DaF-Stunden dramapädagogisch gestalten - wie mache ich das? In: Schlemminger, Gerald / Brysch, Thomas / Schewe, Manfred (eds.): Pädagogische Konzepte für einen ganzheitlichen DaF-Unterricht. Berlin: Cornelsen, 72-105

Schewe, Manfred / Shaw, Peter (eds.) (2003): Towards Drama as a Method in the Foreign Classroom. Frankfurt/M.: Peter Lang

Schimizu, Toyoko (1993): Initial Experiences with Improvised Drama in English Teaching: Working Against the Historical Neglect of Fluency in Language Learning in Japan. In: Schewe, Manfred; Shaw, Peter (eds.): Towards Drama as a Method in the Foreign Classroom. Frankfurt/M.: Peter Lang, 139-169

Schmidt, Richard (1994): Deconstructing consciousness in search of usual definitions for applied linguistics. AILA Review 11, 11-26

Swain, Merrill (1985): Communicative Competence: Some Roles of Comprehensible Input an Comprehensible Output in its Developement. In: Gass, Susan M. / Madden, Carolyn (eds.): Input in Second Language Acquisition. Rowley, Mass.: Newbury House, 235-253 
Stern, Hans Heinrich (1975). What can we learn from the good language learner? Canadian Modern Language Review 31, 304-318

Tönshoff, Wolfgang (1995): Fremdsprachenlerntheorie. Ausgewählte Forschungsergebnisse und Denkanstöße für die Unterrichtspraxis. In: Fremdsprache Deutsch (Sonderheft) 4-15

Wettemann, Ulrich (2006): Welche Lernmethode verspricht den meisten Lernerfolg? In: INFO AMPAL 13/14, 29-30

Wettemann, Ulrich (2004): Schlüsselqualifikationen: Das Ticket für die moderne Arbeitswelt. Eine Einführung in den autonomiefördernden DaF-Unterricht für Mexiko. Masterarbeit in Deutsch als Fremdsprache, Universität Kassel (erscheint März 07:

http://www.grin.com/de/preview/6769.html)

Wolff, Dieter (1994): Konstruktivismus: Ein neues Paradigma in der Fremdsprachendidaktik? In: Die neueren Sprachen 93/3, 407-429

Wolff, Dieter (1999): Zu den Beziehungen zwischen Theorie und Praxis in der Entwicklung von Lernerautonomie. In: Edelhoff, Christoph / Weskamp, Ralf (eds.): Autonomes Fremdsprachenlernen. Ismaning: Hueber 39-48 


\section{A Anhang A:}

\section{A.1 Tabelle 1: Chunks für die unterrichtliche Kommunikation 1}

Tabelle 1: Table 1

\begin{tabular}{|c|}
\hline Wie sagt man ... auf Deutsch? (für Chunks) \\
Was heißt/bedeutet ... (das) auf Spanisch? (für Wörter) \\
Wie bitte? \\
Noch mal bitte. \\
Wie schreibt man das? \\
Ich verstehe (das) nicht. \\
Ist das richtig? \\
$\ldots$ \\
\hline..
\end{tabular}

\section{A.2 Tabelle 2: Information Gap: In der Cafeteria 1}

\section{A.3 Evaluationsbogen (übersetzt)}

1. War es einfach oder schwierig für dich in diesem Kurs Deutsch zu lernen? Kannst du deine Sichtweise an einem oder zwei Beispielen kurz veranschaulichen?

2. Hast du im Unterricht Hilfestellungen bekommen, um leichter zu lernen? Wenn du dieser Meinung bist, könntest du einige Beispiele nennen?

3. Durch die Art und Weise, wie die Sprache in Szenarien angewendet wurde, glaubst du, dass du dir die sprachlichen Mittel besser merken konntest?

4. Hat dich die Form des Unterrichtens und Lernens motiviert? Könntest du dafür ein paar Beispiele bringen?

5. Hattest du im Unterricht die Möglichkeit, deine Kreativität und deine persönlichen Interessen und Bedürfnisse einzubringen? Falls es so war, könntest du bitte einige Beispiele anführen? 
Tabelle 2: Table 2

\begin{tabular}{|l|l|l|}
\hline $\begin{array}{l}\text { Möchtest du Kaffee? Nimmst du } \\
\text { Milch und Zucker? (4) }\end{array}$ & Gleichfalls. (9) \\
\hline Tag Aranza, ist hier noch frei? (7) & & (Einen) Orangensaft, bitte. (6) \\
\hline $\begin{array}{l}\text { Hallo Paul, wie geht's? Nicht so } \\
\text { gut. (1) }\end{array}$ & Gut und Ihnen? (2) \\
\hline $\begin{array}{l}\text { Woher kommst du? Aus } \\
\text { Brasilien. (10) }\end{array}$ & Nein, lieber ein Mineralwasser. (5) \\
\hline Ich muss jetzt gehen. (8) & $\begin{array}{l}\text { Nein, ich komme aus Mexiko. (oder) } \\
\text { Nein, ich bin Mexikaner. (3) }\end{array}$ \\
\hline Möchten Sie Apfelsaft? (5) & $\begin{array}{l}\text { Ja, gerne. Nein, nur Milch/Zucker. } \\
\text { (4) }\end{array}$ \\
\hline Freut mich sehr. (9) & Ja klar, setz dich, Victor. (7) \\
\hline $\begin{array}{l}\text { Guten Tag, Herr Schmidt. Wie } \\
\text { geht es Ihnen? (2) }\end{array}$ & Aus den USA und du? (10) \\
\hline $\begin{array}{l}\text { Was trinkst du? (oder) Was } \\
\text { möchtest du trinken? (6) }\end{array}$ & Danke, gut und dir? (1) ... \\
\hline Kommst du aus der Türkei? (3) & $\begin{array}{l}\text { Oh Gott, ich auch. Es ist schon spät. } \\
\text { (8) }\end{array}$ \\
\hline
\end{tabular}

6. Zu Beginn des Kurses haben wir die Erwartungen von jedem Teilnehmer herausgearbeitet. Wurden deine Erwartungen an den Kurs erfüllt?

7. Würdest du gern mit dieser Art von Unterricht fortfahren oder hättest du andere Vorschläge?

8. Da es nicht deine erste Fremdsprache ist, die du lernst, hast du Lernerfahrungen. Wie war der Unterricht im Vergleich zu deinem bisherigen Unterricht?

9. Wenn der Unterricht immer so wäre...

10. Kommentare und Vorschläge aller Art: 


\section{B Anhang B}

\section{B.1 1. - 6. Dramapädagogische Einheit}

1. Dramapädagogische Einheit: Wie heißt du?

Tabelle 3: Table 3

\begin{tabular}{|c|c|}
\hline $\begin{array}{l}\text { Kommunikative } \\
\text { Ziele: }\end{array}$ & $\begin{array}{l}\text { sich vorstellen, jemanden vorstellen, nach dem Namen } \\
\text { fragen können }\end{array}$ \\
\hline Grammatische Ziele: & (in)formell, Personalpronomen und Konjugationsendung ${ }^{a}$ \\
\hline Input/Chunks: & $\begin{array}{l}\text { Ich heiße Anna. } \\
\text { Er/Sie heißt Paul. } \\
\text { (Und) Wie heißt du? Wie heißen Sie? } \\
\text { Ich heiße Victor. } \\
\ldots\end{array}$ \\
\hline \multicolumn{2}{|c|}{ Weg zur dramapädagogischen Umsetzung (DPU) } \\
\hline Erarbeitungsphase & $\begin{array}{l}\text { Wird an der Tafel notiert, wobei Personalpronomen und } \\
\text { Konjugationsendungen markiert werden und die Intonation } \\
\text { durch Pfeile verdeutlicht. }\end{array}$ \\
\hline $\begin{array}{l}\text { DPU: Stehkreis, } \\
\text { Gummiball. }\end{array}$ & $\begin{array}{l}\text { Lehrer beginnt: stellt sich vor, stellt die Person rechts von sich } \\
\text { vor, wirft den Gummiball und fragt. TN stellt sich vor, stellt die } \\
\text { Person rechts von sich vor, wirft den Gummiball und fragt... }\end{array}$ \\
\hline
\end{tabular}

${ }^{a}$ In der zweiten Woche wurden zur Förderung eines autonomiefördernden Fremdsprachenunterrichts die Konjugationsformen selbständig mit dem Wörterbuch und dem Lehrwerk (Berliner Platz 1) erarbeitet. Die unregelmäßigen Verben werden ebenfalls auf Kärtchen übertragen: Auf die eine Seite die Konjugation, wobei die Endungen und Veränderungen im Stamm markiert werden und auf der anderen Seite 3 Beispielsätze.

2. Dramapädagogische Einheit: Erweiterung zu einem Dialog

3. Dramapädagogische Einheit: Erarbeitung einer fiktiven Rolle

4. Dramapädagogische Einheit: Durch minimalen Kontext ein Szenario schaffen

5. Dramapädagogische Einheit: In der Cafeteria

6. Dramapädagogische Einheit: In der Cafeteria mit Kellner/Fräulein 
Tabelle 4: Table 4

\begin{tabular}{|c|c|}
\hline $\begin{array}{l}\text { Erweiterung der } \\
\text { kommunikativen Ziele: }\end{array}$ & $\begin{array}{l}\text { sich begrüßen und verabschieden, nachfragen: nach } \\
\text { Herkunftsland fragen können }\end{array}$ \\
\hline Input/Chunks: & $\begin{array}{l}\text { Hallo, Tag, - Tschüs (informell) } \\
\text { Guten Tag. - Auf Wiedersehen. (formell) } \\
\text { Woher kommst du? Woher kommen Sie? } \\
\text { Ich komme aus Mexiko. Er/Sie kommt aus ... } \\
\text { Entschuldigung. Wie bitte? } \\
\text { (Freut mich sehr. - Gleichfalls.) }^{a}\end{array}$ \\
\hline \multicolumn{2}{|l|}{ Weg zur DPU } \\
\hline Erarbeitungsphase: & $\begin{array}{l}\text { Die ersten Lernschritte aus der vorhergehenden Einheit } \\
\text { werden nun mit Hilfe von bildunterstützten Minidialogen und } \\
\text { den entsprechenden Hörtexten erweitert. } \\
\text { Erweiterung der Sätze aus Einheit eins zu einem Minidialog } \\
\text { und der Rahmensetzung formell oder informell. } \\
\text { Exkurs: Begrüßung in Mexiko/Deutschland: } \\
\text { Wangenkuss/Händeschütteln? }\end{array}$ \\
\hline \multicolumn{2}{|l|}{$\begin{array}{l}\text { Übungsphase in } \\
\text { Partnerarbeit (PA) }\end{array}$} \\
\hline DPU im Halbkreis: & $\begin{array}{l}\text { Ganzheitliche Umsetzung des Dialoges vor der } \\
\text { Beobachtergruppe. }\end{array}$ \\
\hline $\begin{array}{l}\text { Aufgabe der } \\
\text { Beobachtergruppe } \\
\text { und Eingriffsmöglich- } \\
\text { keiten: }\end{array}$ & $\begin{array}{l}\text { Auf die formelle Richtigkeit achten: Personalpronomen, } \\
\text { Konjugation, Rahmensetzung: (in)formell. Die Beobachter } \\
\text { sagen:,Stopp!, Korrektur:“ und soufflieren den/die } \\
\text { korrekte/n Satz/Form und dann kommt: „Noch mal bitte.“, } \\
\text { damit die richtige Form wiederholt wird. }\end{array}$ \\
\hline
\end{tabular}

${ }^{a}$ Der in Klammern gesetzte Input wurde von den Lernern in ihrer Muttersprache vorgeschlagen und von mir pragmatisch angemessen übertragen. 
Tabelle 5: Table 5

\begin{tabular}{|c|c|}
\hline $\begin{array}{l}\text { Kommunikative } \\
\text { Ziele: }\end{array}$ & $\begin{array}{l}\text { Name, Herkunftsland, Stadt und Sprachkenntnisse nennen } \\
\text { können }\end{array}$ \\
\hline Input: & $\begin{array}{l}\text { Ländernamen: Deutschland, Österreich, die Schweiz, die } \\
\text { USA, Frankreich, England, Spanien, Brasilien, Italien } \\
\text { Städte: Lerner schlagen selbst Städtenahmen vor (Weltkarte) } \\
\text { Sprachen: deutsch, englisch, französisch, spanisch, } \\
\text { portugiesisch, italienisch } \\
\text { Fiktive Personen: Lerner bringen angemessene } \\
\text { Personennamen für jedes Land ein }\end{array}$ \\
\hline Chunks: & $\begin{array}{l}\text { Name: siehe DPE 1; Land: siehe DPE } 2 \\
\text { Ich komme aus Spanien... aus der Schweiz, den USA. } \\
\text { Stadt: Wo wohnst du? Wo wohnen Sie? Ich wohne in ... } \\
\text { Sprachen: Welche Sprachen sprichst du/sprechen Sie? Ich } \\
\text { spreche ... }\end{array}$ \\
\hline \multicolumn{2}{|r|}{ I } \\
\hline Erarbeitungsphase: & $\begin{array}{l}\text { Lerner bauen an der Tafel die Rollen auf: für jedes Land eine } \\
\text { Person mit typischem Namen, ihrer Stadt und } \\
\text { Sprachkenntnissen; Beispiel: Deutschland, Paul Maier, Berlin, } \\
\text { Deutsch, Englisch und Französisch }\end{array}$ \\
\hline Übungsphase: & $\begin{array}{l}\text { Lerner notieren ihre ausgewählte Rolle auf ein Kärtchen: die } \\
\text { entsprechenden Fragen und Antworten werden auf Vorder- } \\
\text { und Rückseite von Kärtchen notiert. In PA befragen sich die } \\
\text { Lerner gegenseitig und identifizieren sich mit der Rolle.Lerner } \\
\text { binden ihre Rolle in Dialoge ein }\end{array}$ \\
\hline $\begin{array}{l}\text { DPU im } \\
\text { Halbsitzkreis } \\
\text { (Beobachterrolle; } \\
\text { siehe DPE davor) }\end{array}$ & $\begin{array}{l}\text { sich vorstellen in der Rolle ganzheitliche dialogische } \\
\text { Umsetzung im Schutz der Rolle Reflexion im Muttersprache } \\
\text { über Befinden; Grund für Bereitschaft für Videoaufnahme }\end{array}$ \\
\hline
\end{tabular}


Tabelle 6: Table 6

\begin{tabular}{|l|l|}
\hline $\begin{array}{l}\text { Kommunikative } \\
\text { Ziele: }\end{array}$ & $\begin{array}{l}\text { weitere Begrüßungsroutinen können auf Aussagen reagieren } \\
\text { können }\end{array}$ \\
\hline $\begin{array}{l}\text { Input/Chunks: } \\
\text { in dieser Einheit } \\
\text { unten) }\end{array}$ & $\begin{array}{l}\text { Begrüßung (formell): Guten Morgen, Guten Tag, Guten } \\
\text { Abend (Vergleich der Tagesdauer) } \\
\text { Begrüßung (informell): Hallo, Hi, Tag }\end{array}$ \\
& $\begin{array}{l}\text { „Reaktionschunks“: Wow, echt, wirklich, schön, gut. } \\
\text { (Ich liebe ... (Französisch). (Paris) ...ist toll.) } \\
\text { (Das ist nicht wahr/ Das kann nicht sein...) }\end{array}$ \\
\hline Weg zur DPU & $\begin{array}{l}\text { Erarbeitung und Erweiterung von Reaktionschunks etc. } \\
\text { (Bewusstmachung von Jugendsprache); } \\
\text { Festlegung der Rahmenkombinationen in Kleingruppen: } \\
\text { formell/informell - am Morgen, Mittag oder Abend } \\
\text { Präsentationsmuster der fiktiven Rolle: } \\
\text { Ich heiße ... und komme aus ... Ich wohne in .... und bin ... } \\
\text { (alt }{ }^{a} \text { ) Ich spreche ... } \\
\text { Feste Formulierung für Ortsangabe und Auswahl für } \\
\text { Gefühlszustand: } \\
\text { Heute beginnt der Deutschkurs in Berlin und ich bin } \\
\text { nervös/gespannt/cool oder müde. }\end{array}$ \\
\hline Uideoaufzeichnung \\
$\begin{array}{l}\text { Mit Hilfe der Schritte, Chunks und Rahmensetzungen, die } \\
\text { allen klar an der Tafel ersichtlich sind, bereiten sich die Lerner } \\
\text { in Partnerarbeit intensiv für die erste Videoaufnahme vor. }\end{array}$ \\
$\begin{array}{l}\text { 1. sich vorstellen in der Rolle } \\
\text { 2. ganzheitliche dialogische Umsetzung im Schutz der Rolle } \\
\text { 3. Abschließendes Anschauen der Videoaufzeichnungen und } \\
\text { Auto-Evaluation mit Hilfe eines Evaluationsbogens. }\end{array}$ \\
\hline
\end{tabular}

${ }^{a}$ Das fiktive Rollenalter sollen die Lerner selbständig aus einer Liste von Zahlwörtern entnehmen. 
Tabelle 7: Table 7

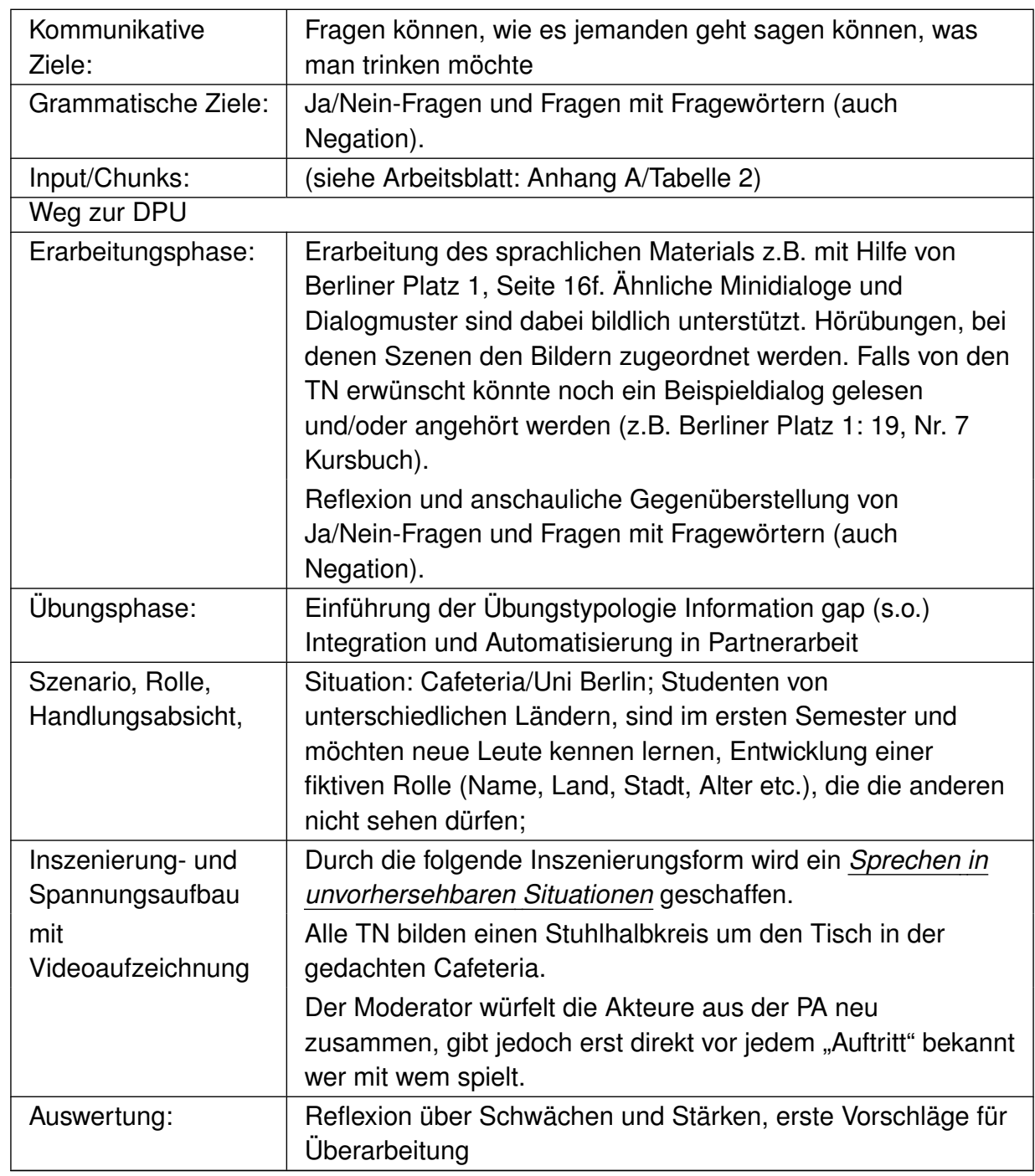


Tabelle 8: Table 8

\begin{tabular}{|l|l|}
\hline $\begin{array}{l}\text { Kommunikative } \\
\text { Ziele: }\end{array}$ & $\begin{array}{l}\text { den Kellner rufen, bestellen und abschließend bezahlen } \\
\text { können; die Zahlen dafür angemessen verwenden können; }\end{array}$ \\
\hline Input/Chunks: & $\begin{array}{l}\text { Was trinkst du? Was nimmst du? Was möchtest du trinken? } \\
\text {.. Ich ... }\end{array}$ \\
\hline & Bezahlen, bitte. Zwei Cola, 3 Kaffee und ein Bier... \\
\hline & Das macht 5 Euro und 30 Cent. Entschuldigung, wie viel? \\
\hline & Vier Euro und siebzig Cent zurück, danke. Danke gleichfalls... \\
\hline Weg zur DPU & $\begin{array}{l}\text { Zahlen durch Vergleich mit Muttersprache }{ }^{a}, \text { Chunks für } \\
\text { Kellner/in direkt (ohne Modelldialog) }\end{array}$ \\
\hline Erarbeitungsphase: & $\begin{array}{l}\text { In der Funktion von Helfer und Kellner arbeiten die } \\
\text { Regisseure weiter an ihrer Szene } \\
\text { Sich in der Rolle vorstellen, Ort und Intention }\end{array}$ \\
\hline Übungsphase: & $\begin{array}{l}\text { Sich begrüßen, um den Platz bitten, sich kennen lernen, } \\
\text { bestellen, bezahlen, sich verabschieden }\end{array}$ \\
\hline DPU: Kontinuum der \\
Inszenierung
\end{tabular}

${ }^{a}$ Der erste Schritt bildet die Entwicklung von NOTICING durch den Vergleich SpanischDeutsch am Beispiel 18: diez y ocho im Gegensatz zu acht - zehn. Zur Förderung dieses Bewusstseins können weitere Übungen herangezogen werden, um die „verdrehte“ Stellung hervorzuheben. Mit Hilfe von Zusatzmaterial können sich die TN die Zahlen selbständig erarbeiten. 